\title{
Trends in the use parenteral nutrition over 10 years: re-audit of referrals to the nutrition support team (NST)
}

\author{
J. Davis and V. Marvin \\ The Royal Surrey County Hospital, Guildford GU2 7XX, UK
}

Timely and appropriate artificial nutrition support and intervention has been shown to reduce length of stay, and, thereby, costs to the $\mathrm{NHS}^{(1)}$.

Parenteral nutrition (PN) is the most expensive form of ready-made artificial nutrition, and should only be used when all other options fail. It requires careful supervision and monitoring and the NST at the Royal Surrey undertake this role auditing the process to encourage the provision of well-planned, clinically effective PN to these 'at risk' patients. In 1996 an audit was undertaken to examine diagnoses, reason for referral and indicators for cessation of $\mathrm{PN}^{(2)}$. This was repeated in 2006 to review recommendations, evaluate if these were being maintained and investigate trends in PN over 10 years. Data were collected retrospectively during the period 1 January-31 December 2006 for 185 PN referrals of which 21 were exclusively ICU, 26 were assigned enteral nutrition, and two had incomplete data:

\begin{tabular}{|l|cc|}
\hline \hline \multicolumn{3}{|c|}{ Diagnosis } \\
\hline & $2006(\%)$ & $1996(\%)$ \\
\cline { 2 - 3 } Upper GI cancer & $30(\mathrm{p}=0.015)$ & 18 \\
Bowel cancer & 12 & 15 \\
Other cancer & 12 & 10 \\
Pancreatitis & 3.5 & 6 \\
IBD & $4(\mathrm{p}=0.007)$ & 13 \\
Other surgical & 32 & 34 \\
Other medical & 6 & \\
\hline
\end{tabular}

$\begin{array}{ll}* \text { Not } & \text { recorded } \\ \text { on } & \text { previous } \\ \text { audit } & \\ & \\ \end{array}$

\begin{tabular}{|lcc|}
\hline \multicolumn{3}{|c|}{ Reasons for stopping PN } \\
\hline & $2006(\%)$ & $1996(\%)$ \\
\cline { 2 - 3 } Eating some (500-1000 kcals) & 28 & 39 \\
Encouraged to eat (<500 kcals) & 16 & 7 \\
Adequate oral intake & 39 & 16 \\
& & $\mathrm{p}<0.001$ \\
Died/Treatment withdrawn & 10 & 12 \\
Line problem, not sepsis & $<1$ & 8 \\
Line related pyrexia & 5 & 4.5 \\
Transfer to other unit & $<1$ & N/A* \\
\hline
\end{tabular}

\begin{tabular}{|lcc|}
\hline \multicolumn{3}{|c|}{ Reasons for referral } \\
\hline & $2006(\%)$ & $1996(\%)$ \\
\cline { 2 - 4 } Unplanned post-op & $18(\mathrm{p}=0.361)$ & 23 \\
PN & & \\
Planned post-op PN & 27 & 21 \\
Obstruction / Ileus & 21 & 18 \\
Gut rest $\quad \pm$ & 20 & 17 \\
malabsorption & & \\
Dysphagia & 8 & 9 \\
Fistula & 2.5 & 5 \\
Planned pre-op PN & $<1$ & 1.5 \\
Other & 2.5 & 1.5 \\
\hline
\end{tabular}

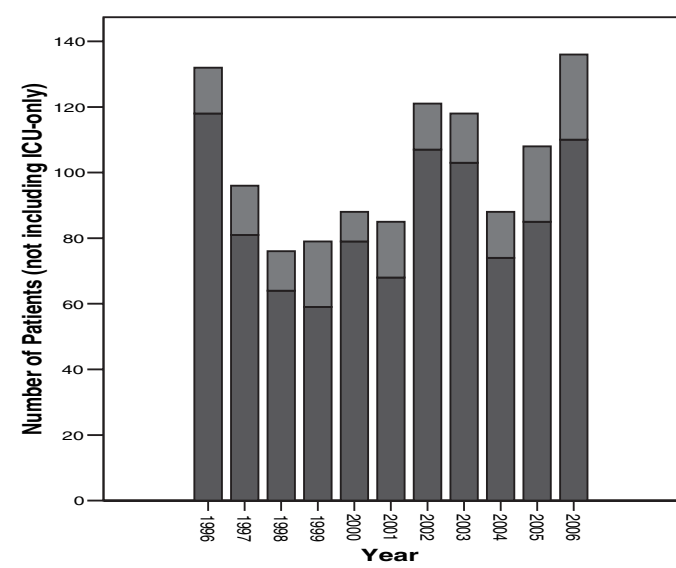

P PN total $\square$ PN not started

In the 10 years, progress has been made; more patients may have received planned PN than previously, and more were assigned for enteral support (1996: $n$ 15, 2006: $n 26$ =0.135) producing a cost saving to the NHS. Enteral and PN feeding policies have been developed and there has been greater specialist dietetic input. The NST has received more structured referrals as an integrated part of planned patient care pathways and baseline biochemistry is now more complete and documented ${ }^{(3)}$. However, although significantly improved since $1996(p<0.001)$, early cessation of PN is still a problem as only $39 \%$ of patients reach adequate intake when PN is stopped.

1. McWhirter JP \& Pennington CR (1994) British Medical Journal, 308, 945-948.

2. Davis J \& Marvin V (1998) Proceedings of the Nutrition Society, $\mathbf{5 7}(\mathbf{3}), 91 \mathrm{~A}$.

3. Marvin V, Livingstone C, May C \& Davis J (2007) Hospital Pharmacist, 14, 166-169. 